\title{
Article
}

\section{Assessment of Lumbar Extensor Muscles in the Context of Trunk Function, a Pilot Study in Healthy Individuals}

\author{
Daniela Vlažná ${ }^{1,2,3} \mathbb{D}$, Peter Krkoška ${ }^{2,3} \mathbb{D}^{\mathbb{D}}$, Matyáš Kuhn ${ }^{4}$, Filip Dosbaba ${ }^{1} \mathbb{D}$, Ladislav Batalik ${ }^{1,5, * \mathbb{D}}$, \\ Eva Vlčková ${ }^{2,3}$, Stanislav Voháňka ${ }^{2,3}$ and Blanka Adamová 2,3 (D) \\ 1 Department of Rehabilitation, University Hospital Brno, 62500 Brno, Czech Republic; \\ Vlazna.Daniela@fnbrno.cz (D.V.); Dosbaba.Filip@fnbrno.cz (F.D.) \\ 2 Department of Neurology, University Hospital Brno, 62500 Brno, Czech Republic; \\ Krkoska.Peter@fnbrno.cz (P.K.); Vlckova.Eva@fnbrno.cz (E.V.); Vohanka.Stanislav@fnbrno.cz (S.V.); \\ Adamova.Blanka@fnbrno.cz (B.A.) \\ 3 Faculty of Medicine, Masaryk University, 62500 Brno, Czech Republic \\ 4 Institute of Biostatistics and Analysis Ltd., 60200 Brno, Czech Republic; kuhn@biostatistika.cz \\ 5 Department of Public Health, Faculty of Medicine, Masaryk University, 62500 Brno, Czech Republic \\ * Correspondence: Batalik.Ladislav@fnbrno.cz
}

\section{check for}

updates

Citation: Vlažná, D.; Krkoška, P.; Kuhn, M.; Dosbaba, F.; Batalik, L.; Vlčková, E.; Voháňka, S.; Adamová, B. Assessment of Lumbar Extensor Muscles in the Context of Trunk Function, a Pilot Study in Healthy Individuals. Appl. Sci. 2021, 11, 9518. https://doi.org/10.3390/ app11209518

Academic Editors: Alessandro de Sire and Ozden Ozyemisci Taskiran

Received: 5 July 2021

Accepted: 12 October 2021

Published: 13 October 202

Publisher's Note: MDPI stays neutral with regard to jurisdictional claims in published maps and institutional affiliations.

Copyright: (c) 2021 by the authors. Licensee MDPI, Basel, Switzerland. This article is an open access article distributed under the terms and conditions of the Creative Commons Attribution (CC BY) license (https:/ / creativecommons.org/licenses/by/ $4.0 /)$.

\begin{abstract}
A comprehensive assessment of trunk function, including the lumbar extensor muscles, appears to be important in various conditions affecting axial musculature. This pilot cross-sectional observational study aimed to define a battery of tests that comprehensively assess trunk muscle function (strength and muscular endurance). Sixty subjects without low back pain (LBP) underwent measurement of isometric lower back extensor strength using a hand-held dynamometer (HHD) in three positions; measurement of respiratory muscle strength; and Biering-Sørensen, prone-plank, and side-bridge tests. The repeatability, short-term and long-term reliability using the HHD device in different postural positions was confirmed. The greatest isometric lower back extensor strength was generated in the sitting position by male subjects. Time of effort in the Biering-Sørensen test was longer in women and older subgroups than in men and younger individuals, although this was not the case for the other two muscular endurance tests. This pilot monitoring of trunk muscle strength and endurance in healthy volunteers may lead to a better understanding of trunk muscle function. Based on this methodological background, the authors aim to use the defined battery of tests in their further studies in a group of patients with LBP and certain neuromuscular diseases to verify its usefulness in clinical practice.
\end{abstract}

Keywords: paraspinal muscles; muscle strength; muscular endurance; function tests; low back pain; neuromuscular diseases

\section{Introduction}

The lumbar extensor muscles, specifically the lumbar paraspinal muscles, comprise two main groups: the transversospinalis group, including the multifidus muscle, and more laterally, the erector spinae muscles, which consist of the longissimus and iliocostalis muscles [1,2]. Trunk stability is maintained by the deep spinal (core) stabilization system, including the lumbar paraspinal muscles, diaphragm, abdominal muscles, and pelvic floor. The lumbar extensor muscular system, more precisely the lumbar multifidus muscle and the erector spinae muscle, plays an essential role in stabilizing the lumbar spine and initiating and controlling all movements in the lumbar vertebral column [3,4]. By controlling these movements, the lumbar extensors also stabilize and protect the underlying osteoligamentous spinal structures from potentially harmful stresses that might otherwise be experienced as a result of movements made beyond their optimal functional range and/or over protracted periods [5]. There is evidence of an association between decreased strength, muscular endurance, atrophy, and excessive fatigability of the lumbar extensors, 
and chronic non-specific low back pain [6-12]. Prospective studies also suggest lumbar extensor deconditioning may be a common risk factor predicting acute low back injury and low back pain (LBP) [11,12].

A gold standard for appropriate quantitative methods of evaluating trunk function (defined as trunk or core stability, including the coordination, strength, and muscular endurance of trunk muscles) has yet to be determined $[13,14]$. However, such an exhaustive assessment appears to be important not only in patients with LBP, but also in patients with certain neuromuscular diseases (NMDs) in which the axial musculature is affected (e.g., axial myopathies, amyotrophic lateral sclerosis, spinal muscular atrophy) [15]. A comprehensive evaluation of trunk function may well be of value in following the patterns of disease progression over time, assisting in selecting appropriate rehabilitation techniques and exercises, and in monitoring the effects of treatment.

Many fields and laboratory protocols have been employed to assess trunk muscle strength and muscular endurance in sports, fitness, clinical, and research settings [16]. Isokinetic dynamometry has been widely used to measure trunk-muscle strength in light of its high validity, reliability, and capacity to measure different muscle groups while controlling contraction type, angular velocity, range of motion, body position, and the number of repetitions and sets [16]. However, high costs, the need for considerable user expertise, and protracted testing time together constitute drawbacks of this approach [17]. In contrast, field tests are easy to perform, do not require extensive and expensive equipment, and allow large numbers of people to be evaluated in a short period [16]. Recently, the use of a hand-held dynamometer (HHD) has been described in the literature for the assessment of lower back extensor strength. This alternative method appears to have great potential, and is quick, inexpensive, and reliable $[15,17,18]$. However, using an HHD to assess isometric lower back extensor strength has not yet been standardized.

This study aimed to create a battery of simple tests that would examine trunk function (strength and muscular endurance) comprehensively, with a special focus on lumbar extensor muscles, to unify the methodology of the tests selected, to confirm the reliability and validity of dynamometric protocols, and to monitor differences in performance between sex and age subgroups in healthy volunteers without chronic LBP in current socioeconomic conditions.

\section{Materials and Methods}

The study was prospective and observational, using test-retest reliability and validation. The local institutional medical research Ethical Committee approved the study protocol (number of the agreement 22-100620/EK), and written and informed consent was obtained from all participants.

\subsection{Participants}

All participants were employees of the University Hospital, including medical doctors, physiotherapists, and nurses. Each subject was interviewed and examined by an experienced neurologist. Exclusion criteria were as follows: age < 18 years, acute low back pain, non-specific chronic low back pain (of duration over 12 weeks) in the medical history, presence of lumbosacral radicular pain in the medical record with residual signs of nerve root dysfunction (sensory impairment and/or absent or diminished reflexes and/or weakness) in clinical neurological examination and manual muscle testing of the lower extremities, previous surgery of the lumbar spine, vertebral fracture, spine infection or tumor, presence of myopathy, comorbid conditions affecting the overall mobility of the patient (e.g., post-stroke paresis, heart failure leading to limited mobility), and confirmed pregnancy.

\subsection{Procedures and Evaluation}

HHD measured maximum isometric lower back extensor strength in three positions: prone, sitting, and standing. As respiratory muscles are also a very important component 
of the core trunk system, maximal strength of inspiratory muscles (maximum inspiratory pressure, MIP) and maximal strength of expiratory muscles (maximum expiratory pressure, $\mathrm{MEP}$ ) were measured using an electronic pressure gauge. Muscular endurance of the trunk muscles was assessed using the Biering-Sørensen test for trunk extensors, by prone-plank test for abdominal core muscles, and by side-bridge test (on both sides) for lateral core muscles.

The examiner administered the short form of the International Physical Activity Questionnaire (IPAQ) to quantify the everyday physical activity of each participant. An experienced physiotherapist recorded all measurements.

The repeatability (test-retest reliability) of measurements obtained by HHD, performed in close succession at the first visit, was assessed in all 60 participants. HHD measurements' short-term and long-term test-retest reliability was evaluated through data from the first visit and the retest visit, which took place either an hour later or two weeks later (details appear below). Any correlation between isometric back extensor strength and respiratory muscle strength of the whole study group was investigated to assess the construct validity of the dynamometry protocol. Mutual correlations between outcomes of all tests in all volunteers were calculated. Further on, the values derived from each test were also analyzed with respect to age and sex to monitor the distribution of data.

\subsection{Dynamometry Protocol}

A hand-held MicroFET 2 (Hoggan Scientific, LLC.) dynamometer was used to measure maximal isometric lower back extensor strength. The measurement range of the device is 0-300 lbs force/660 Newtons / $135 \mathrm{~kg}$. The test was performed in three positions, each following the methodology used in previous studies [17-19].

First, the participant lay on the table in a prone position with legs fixed to the table by a non-elastic belt just above the ankles. The examiner stood next to them, resting their hands with the portable dynamometer on the participant's 7 th thoracic vertebra. The participant was asked to raise the upper body against the examiner's resistance, keeping the arms at the sides of the trunk (Figure 1a). The maximal isometric lower back extensor strength in the prone position was recorded.

Next, the participant was seated on a purpose-designed chair that prevented the feet from reaching the floor. Each participant was asked to sit up straight, and the pelvis was bound by a non-elastic belt to the chair, $1 \mathrm{~cm}$ below the anterior superior iliac spine so that the pelvis was fixed and could not be moved throughout the test. The participant was then asked to cross their arms over the chest and bend forward so that the portable dynamometer could be placed at the level of the 7th thoracic vertebra. After that, the participant was asked to push back as hard as possible (Figure 1b). The maximal isometric lower back extensor strength in the sitting position was recorded.

The participant was then asked to stand back against a wall between two vertical anchor rails. Both feet were placed on the floor, heels together, touching the wall. The pelvis was fixed to the wall by a non-elastic belt looped through the anchor rails and tracing a line $1 \mathrm{~cm}$ below the anterior superior iliac spine so that the pelvis was fixed tight and could not be moved throughout the test. The participant was asked to cross their arms over the chest and bend forward so that the portable dynamometer could be placed at the level of the 7th thoracic vertebra. After that, the participant was asked to push back as hard as possible (Figure 1c). The maximal isometric lower back extensor strength in a standing position was recorded.

In each position, the participant had six attempts, with $20 \mathrm{~s}$ between them to rest. The result of every attempt was recorded to clarify the repeatability of the testing procedures in all 60 participants. To demonstrate the short-term and long-term test-retest reliability of the strength-testing procedures using HHD, participants who agreed to be retested (49 participants) were randomly divided into two groups by drawing a ticket. One group was asked to come back after an hour for retest of maximal isometric lower back extensor strength (24 participants) and the other group in two weeks (25 participants). 


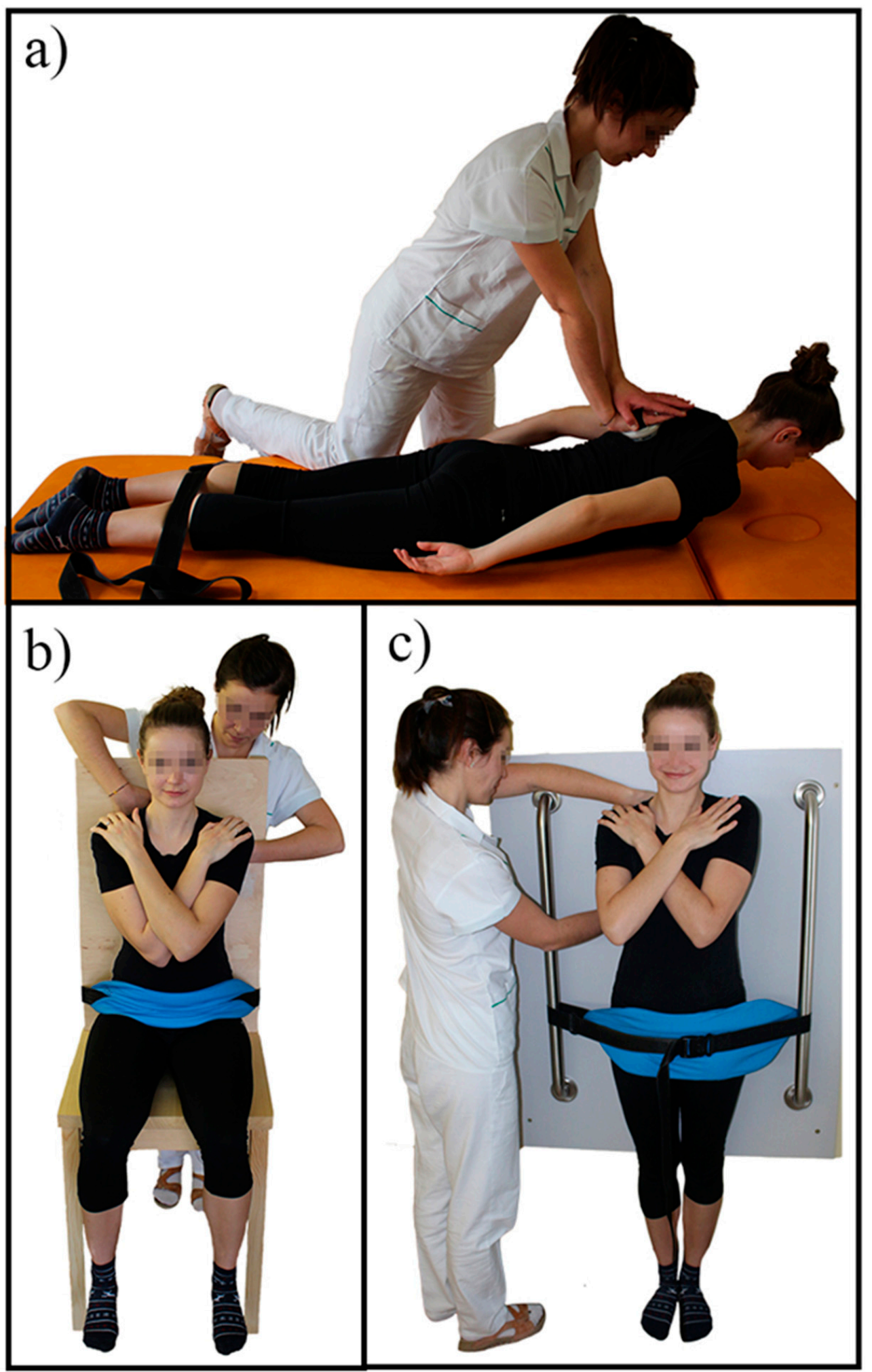

Figure 1. Measurement of maximal isometric lower back extensor strength using HHD (a) in the prone position $(\mathbf{b})$ in the sitting position and $(\mathbf{c})$ in the standing position.

\subsection{Maximal Inspiratory and Expiratory Pressure Protocol}

A microRPM (Micro Medical, Kent, UK) electronic pressure gauge was employed to measure MIP and MEP. The participant was asked to sit straight, and a nose clip was put in place. For measurement of MIP, the participant was asked to exhale completely. The mouthpiece was then inserted, and the subject asked to suck in as hard as possible 
for at least $1.5 \mathrm{~s}$. The highest negative pressure was recorded in centimeters of the water column $\left(\mathrm{cmH}_{2} \mathrm{O}\right)$. For the measurement of MEP, the participant was asked to inhale completely and to blow into the mouthpiece as hard as possible for at least $1.5 \mathrm{~s}$. The most significant positive pressure was recorded in centimeters of the water column $\left(\mathrm{cmH}_{2} \mathrm{O}\right)$. Each measurement (MIP and MEP) was repeated five times in succession, with resting periods of one minute between attempts. Compensatory trunk flexion or extension was not permitted during either of the tests. Both protocols, measuring MIP and MEP, followed a methodology published previously [14].

\subsection{Biering-Sørensen Protocol}

The protocol followed a previously established methodology [14]. The participant was asked to lie in a prone position over the edge of the table, set at the anterior superior iliac spine level. The examiner leaned over the participant to stabilize the lower limbs and pelvis at the ankles and lower calves. The participant was then asked to cross the arms over the chest, then raise the upper part of the body (head, chest, and trunk) so that the trunk was straight. A stopwatch was used to record time between the beginning (raising of the trunk) to the end of the test when the participant was becoming tired, when the trunk was lowering from the horizontal position, or when the arms were stretched over a chair in front of the table. The effort time was recorded in seconds.

\subsection{Prone-Plank Test and Side-Bridge Test Protocol}

At the beginning of the prone-plank test, the participant was asked to lie down in a prone position on a firm mat. The task was to lift the body weight onto the toes and forearms with elbows under shoulders, scapulae adducted, hips at the same level as the spine. For the side-bridge test, the participant was asked to lie on one side on the same firm mat. The legs were to be extended, the lower forearm with the elbow flexed to $90^{\circ}$, the upper arm crossed over the chest. The participant was asked to raise the hips off the mat, holding the position in a horizontal line with the body on a flexed elbow, which was immediately under the shoulder. The test was performed on both sides. During the two tests, prone-plank and side-bridge, the participant was given feedback from the examiner with respect to posture. The test ended when fatigue was evident, and the participant could no longer maintain the correct position. The time taken by the effort was recorded by stopwatch in seconds. Both protocols, prone-plank test and side-bridge test, followed previously established methodology [14].

\subsection{International Physical Activity Questionnaire}

The short form of the IPAQ consisted of three items assessing levels of physical activity and one regarding the amount of time spent in a sitting position. The examiner administered all questionnaire items using a language validated version in the mother tongue of participants so that each participant could understand them equally and answer truthfully [20]. Each question was to be answered in terms of the frequency of activity per week and time spent in activity in one session. These data were used to calculate an outcome, expressed as overall MET-min per week (METs are multiples of the resting metabolic rate) and as one of three levels of physical activity: 1-Low, 2-Moderate, and 3-High [21].

\subsection{Statistical Analysis}

Standard descriptive statistics were applied in the analysis. Continuous variables were described by mean and standard deviation. Categorical variables were characterized by absolute and relative frequencies. The normality of data was checked and all the variables followed the normal distribution. Repeatability, short-term, and long-term test-retest reliability of tests assessing maximal isometric back extensor strength was examined by means of the intra-class correlation coefficient (ICC) derived from a two-way random model with single measures and absolute agreement using R-package IRR for intra-rater 
reliability. The statistical significance of the differences between measurements at different visits was analyzed by means of the paired t-test. All statistical tests used herein are suitable tools for evaluating a group of this size. Correlations between particular strength and muscular endurance tests were computed using Spearman's correlation coefficient $(r s)$ and its corresponding $p$-value. By convention, an $r s$ value higher than 0.80 indicated a robust correlation, an $r s$ value between 0.60 and 0.79 a strong correlation, an $r s$ between 0.40 and 0.59 a moderate correlation, between 0.20 and 0.39 a weak correlation, and an $r s$ below 0.19 meant a very weak correlation [22]. The two-way ANOVA tested differences in performance between sex and age subgroups with posthoc analysis, a test specified to analyze a group of this size. The analysis was performed in R Statistical Software version 3.6.3 (R Core Team 2020) in RStudio (RStudio Team 2020).

\section{Results}

\subsection{Study Subjects}

Sixty volunteers ( 30 male, 30 female) were enrolled in the study with a median age of 35 years. It may be assumed that outcomes recorded should depend on age and sex. Categories were therefore created with respect to age: $18-29$ years, $30-49$ years, $\geq 50$ years, each for male and female (altogether six categories). The categories appear in detail in Table 1.

Table 1. Characteristics of subjects in all subgroups.

\begin{tabular}{|c|c|c|c|c|c|c|}
\hline & Variable & Sex & Total & 18-29 Years & 30-49 Years & $\geq 50$ Years \\
\hline \multirow{2}{*}{\multicolumn{2}{|c|}{$\operatorname{Sex}(n)$}} & Male & 30 & 10 & 15 & 5 \\
\hline & & Female & 30 & 10 & 15 & 5 \\
\hline \multirow{2}{*}{\multicolumn{2}{|c|}{ Age (years) }} & Male & $35.8 \pm 11.7$ & $26.9 \pm 1.4$ & $34.4 \pm 3.7$ & $57.6 \pm 11.5$ \\
\hline & & Female & $38.4 \pm 11.1$ & $26.6 \pm 1.4$ & $40.2 \pm 4.6$ & $56.6 \pm 6.4$ \\
\hline \multirow{2}{*}{\multicolumn{2}{|c|}{ Height (cm) }} & Male & $182.9 \pm 8.3$ & $183.8 \pm 7.2$ & $183.0 \pm 8.5$ & $180.8 \pm 10.9$ \\
\hline & & Female & $167.7 \pm 6.3$ & $170.4 \pm 5.8$ & $166.4 \pm 6.3$ & $166.0 \pm 6.5$ \\
\hline \multirow{2}{*}{\multicolumn{2}{|c|}{ Weight (kg) }} & Male & $81.9 \pm 8.8$ & $82.0 \pm 8.0$ & $82.8 \pm 10.1$ & $79.0 \pm 6.8$ \\
\hline & & Female & $62.2 \pm 6.8$ & $61.1 \pm 5.7$ & $62.3 \pm 7.4$ & $64.0 \pm 8.2$ \\
\hline \multirow{2}{*}{\multicolumn{2}{|c|}{$\operatorname{BMI}\left(\mathrm{kg} / \mathrm{m}^{2}\right)$}} & Male & $24.5 \pm 2.4$ & $24.4 \pm 2.8$ & $24.7 \pm 2.6$ & $24.2 \pm 1.4$ \\
\hline & & Female & $22.1 \pm 2.0$ & $21.0 \pm 1.5$ & $22.5 \pm 2.2$ & $23.2 \pm 1.7$ \\
\hline \multirow{6}{*}{ Education } & \multirow{2}{*}{ Primary education $(n(\%))$} & Male & $0(0)$ & $0(0)$ & $0(0)$ & $0(0)$ \\
\hline & & Female & $1(3.3)$ & $0(0)$ & $0(0)$ & $1(20.0)$ \\
\hline & \multirow{2}{*}{ Secondary education $(n(\%))$} & Male & $1(3.3)$ & $1(10.0)$ & $0(0)$ & $0(0)$ \\
\hline & & Female & $10(33.3)$ & $1(10.0)$ & $7(46.7)$ & $2(40.0)$ \\
\hline & \multirow{2}{*}{ Tertiary education $(n(\%))$} & Male & $29(96.7)$ & $9(90.0)$ & $15(100.0)$ & $5(100.0)$ \\
\hline & & Female & $19(63.3)$ & $9(90.0)$ & $8(53.3)$ & $2(40.0)$ \\
\hline
\end{tabular}

Data are expressed as mean \pm standard deviation. $n$ : number of individuals; BMI: body mass index; (\%) percentage of individuals from a certain category men/women; Primary education: graduated from elementary school; Secondary education: graduated from secondary school with state exam; Tertiary education: graduated from university.

\subsection{Maximal Isometric Lower Back Extensor Strength}

Table 2 shows the values obtained for maximal isometric lower back extensor strength (from the 2nd to the 5th attempt) in all three postural positions measured in the first session for all subjects and for individual categories. The highest values of maximal isometric lower back extensor strength were measured in a sitting position, followed by values measured standing. The lowest values were measured in the prone position.

A significant difference in the values measured appeared between the 1st and 2nd attempts made by men $\geq 50$ years (data not shown). To avoid any potential distortion arising out of the learning effect or fatigue (even though neither of these were demonstrated), values measured at the first and the last attempts in all measurements were excluded. 
Subsequently, the means of values measured from the 2nd to 5th attempts were used for calculation.

Table 2. Maximal isometric lower back extensor strength: results.

\begin{tabular}{|c|c|c|c|c|c|c|c|}
\hline Variable & Sex & Total & 18-29 Years & 30-49 Years & $\geq 50$ Years & $p$-Value Age & $p$-Value Sex \\
\hline \multirow{3}{*}{ Prone position ${ }^{\dagger}$} & Male & $25.7 \pm 5.0$ & $28.5 \pm 4.1$ & $25.6 \pm 3.9$ & $20.5 \pm 6.0$ & \multirow{3}{*}{0.002} & \multirow{3}{*}{0.319} \\
\hline & Female & $24.4 \pm 5.6$ & $26.1 \pm 5.8$ & $24.8 \pm 5.5$ & $20.2 \pm 3.5$ & & \\
\hline & Post-hc & c test (age) & $\mathrm{b}$ & $\mathrm{b}$ & a & & \\
\hline \multirow{2}{*}{ Sitting position $^{\dagger}$} & Male & $80.0 \pm 18.0$ & $78.9 \pm 23.1$ & $83.4 \pm 15.8$ & $72.1 \pm 12.5$ & \multirow{2}{*}{0.066} & \multirow{2}{*}{$<0.001$} \\
\hline & Female & $55.9 \pm 17.8$ & $62.2 \pm 18.3$ & $57.0 \pm 16.2$ & $39.9 \pm 14.0$ & & \\
\hline \multirow{2}{*}{ Standing position ${ }^{\dagger}$} & Male & $62.0 \pm 13.9$ & $61.9 \pm 13.4$ & $64.6 \pm 15.2$ & $54.6 \pm 9.0$ & \multirow{2}{*}{0.074} & \multirow{2}{*}{$<0.001$} \\
\hline & Female & $43.1 \pm 12.6$ & $47.9 \pm 12.2$ & $43.1 \pm 11.6$ & $33.3 \pm 13.0$ & & \\
\hline
\end{tabular}

Data are expressed as mean \pm standard deviation. Post-hoc tests: $a, b-$ same letters marking values of categories within given row denote mutually statistically not different groups. ${ }^{\dagger}$ Maximal isometric lower back extensor strength (calculated from the mean value of the 2 nd to 5th attempts), results expressed in kilograms.

The consistency of the data, expressed as the repeatability of measurements in one session, was excellent (ICC 0.975-0.996) for all six successive attempts and the 2nd to 5th attempts in all three postural positions (Table 3).

Table 3. Maximal isometric lower back extensor strength: repeatability.

\begin{tabular}{|c|c|c|c|}
\hline & Characteristics & All Attempts ${ }^{+}$ & 2nd-5th Attempt ++ \\
\hline $\begin{array}{c}\text { Number of participants } \\
\text { evaluated }\end{array}$ & $n$ & 60 & 60 \\
\hline Prone position & $\begin{array}{c}\text { ICC }(95 \% \text { CI }) \\
p \text {-value }\end{array}$ & $\begin{array}{c}0.978(0.968-0.986) \\
<0.001\end{array}$ & $\begin{array}{c}0.975(0.963-0.984) \\
<0.001\end{array}$ \\
\hline Sitting position & $\begin{array}{c}\text { ICC }(95 \% \mathrm{CI}) \\
p \text {-value }\end{array}$ & $\begin{array}{c}0.995(0.992-0.997) \\
<0.001\end{array}$ & $\begin{array}{c}0.995(0.992-0.997) \\
<0.001\end{array}$ \\
\hline Standing position & $\begin{array}{c}\text { ICC }(95 \% \text { CI }) \\
p \text {-value }\end{array}$ & $\begin{array}{c}0.996(0.994-0.997) \\
<0.001\end{array}$ & $\begin{array}{c}0.996(0.994-0.997) \\
<0.001\end{array}$ \\
\hline
\end{tabular}

$n$ : number of individuals; ICC: intraclass correlation coefficient; CI: confidence interval. ${ }^{\dagger}$ repeatability of measurements at the first visit for all 6 attempts. ${ }^{+\dagger}$ repeatability of measurements at the first visit for the 2 nd to 5 th attempts.

Both short-term and long-term test-retest reliability emerged as excellent in all three techniques measuring maximal isometric lower back extensor strength: ICC varied from 0.937 to 0.992 (Table 4). The difference between values measured at the first and the second visit was not clinically significant.

The strength of lower back extensors was significantly lower in the age category $\geq 50$ years in the prone position. The same tendency was evident in the standing and sitting positions. However, the difference was not statistically significant. The lower back extensor strength in sitting and standing positions was significantly higher in men than in women (Table 2).

\subsection{Maximum Inspiratory and Expiratory Pressure}

Results appear in Table 5. In certain groups, significant differences in values measured between the 1st and the 2nd attempts and between the 4 th and the 5 th attempts emerged (data not shown). The mean of the values measured at the 2nd to 4 th attempt was, therefore, calculated. In the course of measurements of maximum inspiratory pressure, men were able to attain on average $91.0 \%$ and women $95.8 \%$ of the predicted value. For the maximum expiratory pressure, men reached an average at $70.9 \%$ and women $81.3 \%$ of the expected value. There was a statistically significant difference in absolute values measured in men and women, where men reached higher maximum inspiratory and expiratory pressure than women. There was no significant difference seen between age subgroups in either MIP or MEP. 
Table 4. Maximal isometric lower back extensor strength: short-term and long-term test-retest reliability.

\begin{tabular}{|c|c|c|c|}
\hline & Characteristics & Short-Term $^{\dagger}$ & Long-Term ${ }^{\dagger+}$ \\
\hline Number of participants evaluated & $n$ & 24 & 25 \\
\hline Prone position & $\begin{array}{c}\text { ICC }(95 \% \text { CI }) \\
p \text {-value }(\mathrm{ICC}) \\
\text { Mean } \pm \mathrm{SD}^{+++} \\
p \text {-value (t-test) }\end{array}$ & $\begin{array}{c}0.992(0.982-0.997) \\
<0.001 \\
0.0 \pm 0.7 \\
0.855\end{array}$ & $\begin{array}{c}0.937(0.717-0.979) \\
<0.001 \\
-1.1 \pm 1.3 \\
<0.001\end{array}$ \\
\hline Sitting position & $\begin{array}{c}\text { ICC }(95 \% \text { CI }) \\
p \text {-value }(\mathrm{ICC}) \\
\text { Mean } \pm \mathrm{SD}^{+++} \\
p \text {-value (t-test) }\end{array}$ & $\begin{array}{c}0.990(0.977-0.996) \\
<0.001 \\
0.5 \pm 3.0 \\
0.388\end{array}$ & $\begin{array}{c}0.990(0.978-0.996) \\
<0.001 \\
-0.7 \pm 2.9 \\
0.232\end{array}$ \\
\hline Standing position & $\begin{array}{c}\text { ICC }(95 \% \text { CI }) \\
p \text {-value }(\mathrm{ICC}) \\
\text { Mean } \pm \text { SD } \\
p \text {-value (t-test) }\end{array}$ & $\begin{array}{c}0.991(0.981-0.996) \\
<0.001 \\
-0.4 \pm 2.3 \\
0.369\end{array}$ & $\begin{array}{c}0.984(0.962-0.993) \\
<0.001 \\
-1.0 \pm 2.4 \\
0.047\end{array}$ \\
\hline
\end{tabular}

$n$ : number of individuals; ICC: intraclass correlation coefficient; CI: confidence interval; SD: standard deviation. ${ }^{\dagger}$ short-term repeatability of measurements assessed at the first visit and after an hour. ${ }^{+t}$ long-term repeatability of measurements assessed at the first visit and after two weeks. ${ }^{++t}$ difference between values of isometric lower back extensor strength measured at the first and the second visit, expressed in kilograms.

Table 5. Respiratory muscle strength: results.

\begin{tabular}{|c|c|c|c|c|c|c|c|}
\hline Variable & Sex & Total & 18-29 Years & 30-49 Years & $\geq 50$ Years & $p$-Value Age & $p$-Value Sex \\
\hline $\operatorname{MIP}\left(\mathrm{cmH}_{2} \mathrm{O}\right)^{\dagger}$ & $\begin{array}{c}\text { Male } \\
\text { Female }\end{array}$ & $\begin{array}{c}112.4 \pm 19.7 \\
82.1 \pm 22.2\end{array}$ & $\begin{array}{c}115.8 \pm 10.9 \\
75.0 \pm 23.4\end{array}$ & $\begin{array}{c}112.7 \pm 19.2 \\
84.9 \pm 20.2\end{array}$ & $\begin{array}{c}104.9 \pm 34.0 \\
87.8 \pm 26.7\end{array}$ & 0.849 & $<0.001$ \\
\hline $\begin{array}{l}\text { MIP (percentage of } \\
\text { predicted value) }^{\dagger}\end{array}$ & $\begin{array}{l}\text { Male } \\
\text { Female }\end{array}$ & $\begin{array}{l}91.0 \pm 15.1 \\
95.8 \pm 25.8\end{array}$ & $\begin{array}{c}93.4 \pm 8.8 \\
86.2 \pm 26.9\end{array}$ & $\begin{array}{l}90.9 \pm 15.5 \\
97.6 \pm 23.2\end{array}$ & $\begin{array}{c}86.8 \pm 24.7 \\
109.5 \pm 28.4\end{array}$ & 0.573 & 0.393 \\
\hline $\operatorname{MEP}\left(\mathrm{cmH}_{2} \mathrm{O}\right)^{+}$ & $\begin{array}{c}\text { Male } \\
\text { Female }\end{array}$ & $\begin{array}{l}164.3 \pm 30.3 \\
122.8 \pm 30.6\end{array}$ & $\begin{array}{l}157.0 \pm 31.7 \\
115.7 \pm 19.5\end{array}$ & $\begin{array}{l}166.9 \pm 27.8 \\
126.6 \pm 28.3\end{array}$ & $\begin{array}{l}170.9 \pm 38.1 \\
125.7 \pm 54.4\end{array}$ & 0.439 & $<0.001$ \\
\hline $\begin{array}{l}\mathrm{MEP} \text { (percentage of } \\
\text { predicted value) }^{+}\end{array}$ & $\begin{array}{l}\text { Male } \\
\text { Female }\end{array}$ & $\begin{array}{l}70.9 \pm 12.5 \\
81.3 \pm 20.3\end{array}$ & $\begin{array}{l}67.4 \pm 13.6 \\
76.1 \pm 12.8\end{array}$ & $\begin{array}{l}71.6 \pm 11.9 \\
83.3 \pm 18.6\end{array}$ & $\begin{array}{l}76.0 \pm 12.5 \\
86.0 \pm 36.1\end{array}$ & 0.314 & 0.020 \\
\hline
\end{tabular}

Data are expressed as mean \pm standard deviation. MIP: maximum inspiratory pressure; MEP: maximum expiratory pressure. ${ }^{\dagger}$ respiratory muscle strength calculated from the mean value of the 2nd to 4 th attempts.

\subsection{Muscular Endurance of Trunk Muscles}

Outcomes of trunk muscular endurance tests appear in Table 6. The median holding time in the Biering-Sørensen test was greater in women than in men. Subjects under 30 years of age expended significantly less effort than older subjects. The mean time of effort in the prone-plank test was greater in men than in women, although it did not reach statistical significance. No significant difference in time of effort in the prone-plank test appeared among age categories. No significant difference between sexes and age sub-groups could be observed in the mean time effort in the side-bridge test on the left side. However, a distinction was evident between age groups in outcomes for the side-bridge test on the right side, where the age group $\geq 50$ years returned the lowest time of effort.

\subsection{Physical Activity}

The values of physical activity performed per week by the study group appear in Table 7. Most of the volunteers (75\%) undertook a moderate level of physical activity, with only a few individuals scoring high (17\%) or low ( $8 \%)$ levels. The median time spent sitting was six hours a day. 
Table 6. Trunk muscular endurance tests: results.

\begin{tabular}{|c|c|c|c|c|c|c|c|}
\hline Variable & Sex & Total & 18-29 Years & 30-49 Years & $\geq 50$ Years & $p$-Value Age & $p$-Value Sex \\
\hline \multirow{3}{*}{$\begin{array}{l}\text { Biering-Sørensen test } \\
\text { (time (s)) }\end{array}$} & Male & $149.7 \pm 41.3$ & $118.6 \pm 18.5$ & $166.7 \pm 42.7$ & $160.8 \pm 39.5$ & \multirow{3}{*}{$<0.001$} & \multirow{3}{*}{0.025} \\
\hline & Female & $179.8 \pm 61.5$ & $152.7 \pm 30.4$ & $190.3 \pm 67.7$ & $202.4 \pm 79.9$ & & \\
\hline & \multicolumn{2}{|c|}{ Post-hoc test (age) } & $\mathrm{a}$ & $\mathrm{b}$ & $\mathrm{b}$ & & \\
\hline \multirow{2}{*}{$\begin{array}{l}\text { Prone-plank test (time } \\
(\mathrm{s}))\end{array}$} & Male & $165.0 \pm 59.3$ & $163.6 \pm 56.6$ & $165.5 \pm 64.4$ & $166.2 \pm 61.2$ & \multirow{2}{*}{0.971} & \multirow{2}{*}{0.101} \\
\hline & Female & $141.0 \pm 50.0$ & $137.4 \pm 19.4$ & $142.8 \pm 61.2$ & $142.6 \pm 64.0$ & & \\
\hline \multirow{3}{*}{$\begin{array}{l}\text { Side-bridge test—right } \\
\text { side (time (s)) }\end{array}$} & Male & $77.7 \pm 25.4$ & $79.3 \pm 28.4$ & $79.5 \pm 22.2$ & $69.2 \pm 32.4$ & \multirow{3}{*}{0.037} & \multirow{3}{*}{0.676} \\
\hline & Female & $74.9 \pm 28.3$ & $80.1 \pm 24.6$ & $81.8 \pm 27.3$ & $44.0 \pm 20.6$ & & \\
\hline & \multicolumn{2}{|c|}{ Post-hoc test (age) } & $a b$ & $\mathrm{~b}$ & $\mathrm{a}$ & & \\
\hline \multirow{2}{*}{$\begin{array}{l}\text { Side-bridge test-left } \\
\text { side (time (s)) }\end{array}$} & Male & $75.3 \pm 26.5$ & $79.2 \pm 27.9$ & $75.3 \pm 22.7$ & $67.4 \pm 37.9$ & \multirow{2}{*}{0.120} & \multirow{2}{*}{0.822} \\
\hline & Female & $73.6 \pm 32.5$ & $79.7 \pm 24.6$ & $78.5 \pm 37.0$ & $46.6 \pm 20.9$ & & \\
\hline
\end{tabular}

Data are expressed as mean \pm standard deviation. Post-hoc tests: $a$, $b$ —same letters marking values of categories within given row denote mutually statistically not different groups.

Table 7. International Physical Activity Questionnaire, short form: results.

\begin{tabular}{|c|c|c|c|c|c|c|}
\hline \multicolumn{2}{|c|}{ Variable } & Sex & Total & 18-29 Years & 30-49 Years & $\geq 50$ Years \\
\hline \multirow{6}{*}{$\begin{array}{l}\text { Level of physical } \\
\text { activity }\end{array}$} & \multirow{2}{*}{ 1. Low $(n(\%))$} & Male & $1(3.3)$ & $0(0)$ & $0(0)$ & $1(20.0)$ \\
\hline & & Female & $4(13.3)$ & $1(10.0)$ & $2(13.3)$ & $1(20.0)$ \\
\hline & \multirow{2}{*}{ 2. Moderate $[n(\%)]$} & Male & $23(76.7)$ & $9(90.0)$ & $12(80.0)$ & $2(40.0)$ \\
\hline & & Female & $22(73.3)$ & $8(80.0)$ & $12(80.0)$ & $2(40.0)$ \\
\hline & \multirow{2}{*}{ 3. High $[n(\%)]$} & Male & $6(20.0)$ & $1(10.0)$ & $3(20.0)$ & $2(40.0)$ \\
\hline & & Female & $4(13.3)$ & $1(10.0)$ & $1(6.7)$ & $2(40.0)$ \\
\hline \multirow{2}{*}{\multicolumn{2}{|c|}{ MET (minutes per week) }} & Male & $2446.6 \pm 1396.3$ & $2108.3 \pm 1080.4$ & $2461.0 \pm 1156.2$ & $3080.2 \pm 2457.8$ \\
\hline & & Female & $2153.1 \pm 1522.1$ & $1610.1 \pm 1020.8$ & $2183.8 \pm 1073.9$ & $3147.0 \pm 2905.2$ \\
\hline \multirow{2}{*}{\multicolumn{2}{|c|}{ Hours spent sitting (per day) }} & Male & $7.6 \pm 3.2$ & $8.3 \pm 2.7$ & $8.0 \pm 3.2$ & $4.9 \pm 3.4$ \\
\hline & & Female & $5.7 \pm 2.6$ & $7.4 \pm 2.6$ & $4.7 \pm 2.2$ & $5.1 \pm 2.1$ \\
\hline
\end{tabular}

Data are expressed as mean \pm standard deviation. $n$ : number of individuals, (\%) percentage of individuals from certain category men/women; MET: multiple of resting metabolic rate.

\subsection{Correlations between Tests}

A statistically significant correlation between lower back extensor strength measured in prone, sitting, and standing positions was disclosed (Figure 2). However, the correlation was strongest between values measured in sitting and standing positions $(r s=0.83$, $p<0.001)$. A strong correlation between maximum inspiratory pressure and maximum expiratory pressure $(r s=0.78, p<0.001)$ was also evident. Spearman's correlation coefficients evaluating the relation between inspiratory and expiratory muscle strength and lower back extensor strength measured in sitting and standing positions reached 0.49-0.51 $(p<0.001)$. A robust and significant correlation was found between outcomes obtained in side-bridge tests performed on the left and right sides $(r s=0.86, p<0.001)$. The results of the Biering-Sørensen test revealed a moderate correlation with the results of the prone-plank test $(r s=0.41, p=0.01)$. Correlations between outcomes of strength testing procedures and muscular endurance tests were weak or absent. No element of strength and endurance performance correlated significantly with either the level of physical activity or with hours spent per day in a sitting position. 


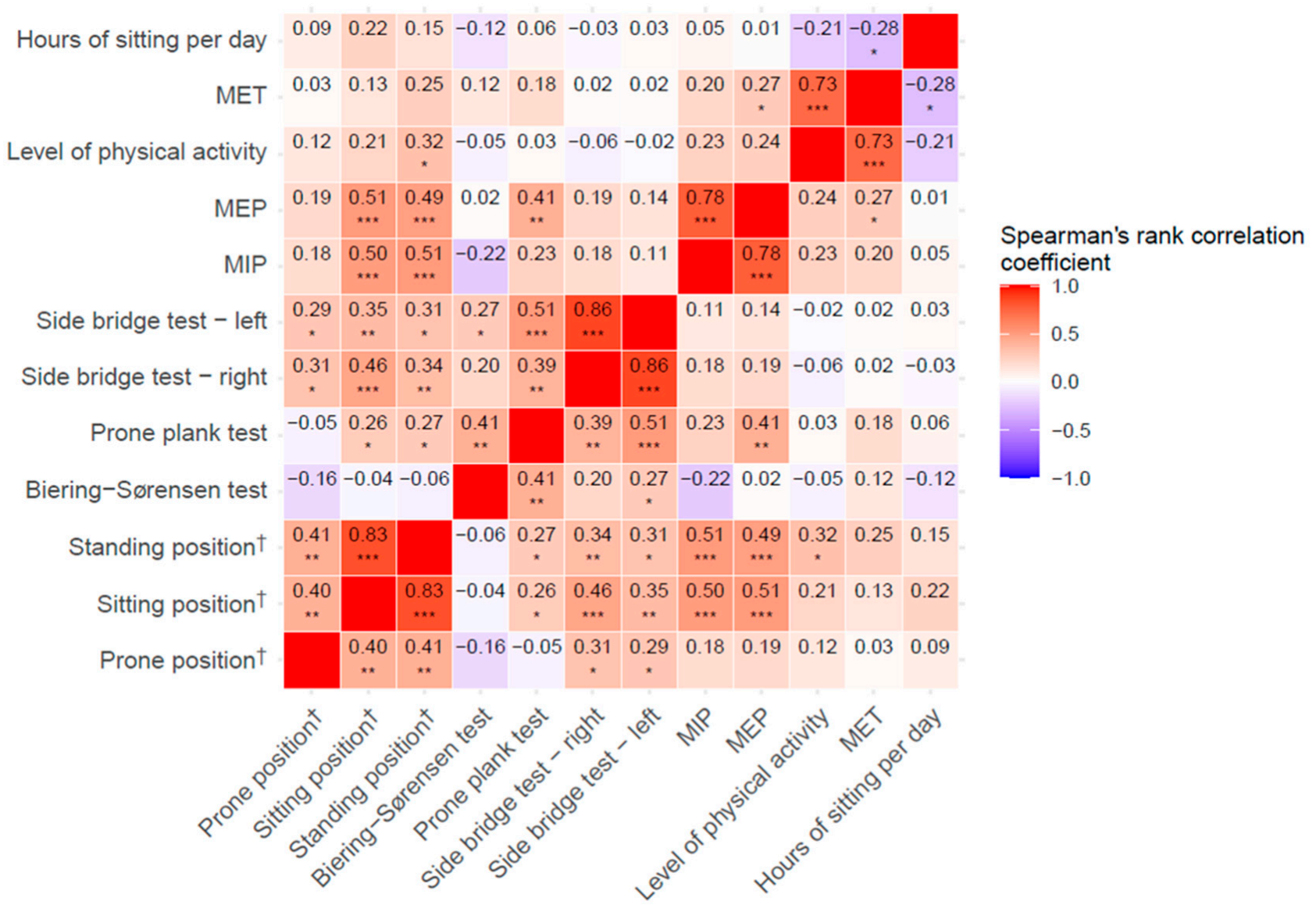

Figure 2. Correlation between strength testing procedures, muscular endurance tests, and IPAQ. Significance of correlations is expressed through asterisks at levels as follows: ${ }^{*} p<0.05,{ }^{* *} p<0.01$, and ${ }^{* * *} p<0.001$. ${ }^{\dagger}$ Maximal isometric lower back extensor strength in different positions.

\section{Discussion}

This study has defined a battery of simple tests that are easy to perform in everyday practice and provide a comprehensive overview of essential components of trunk function (strength and muscular endurance). Strength-testing procedures included measurement of isometric lower back extensor strength using HHD and measurement of respiratory muscle strength. Muscular endurance of the trunk muscles was evaluated by the Biering-Sørensen, prone-plank, and side-bridge tests.

Isokinetic dynamometry is often referred to as the "gold standard" for the measurement of trunk muscle strength $[12,15,16,18]$. A certain number of studies have already been devoted to finding an alternative, practical, cheaper, and more user-friendly clinical tool that might provide reliable information about back extensor strength using HHD. Similar to the current study, the above studies established excellent validity and reliability for the new method [17-19]. The present study confirmed the repeatability and the short-term and long-term test-retest reliability of techniques measuring maximal isometric lower back extensor strength by HHD as excellent in all three positions. Unifying the methodology of the three existing protocols [17-19] and proving the repeatability of measurements using one dynamometer device allows us to compare obtained data measured in different postural positions and to discuss which of the positions is the most reasonable to use in terms of the biomechanics of the motion and in terms of usefulness in everyday practice.

The highest values of isometric lower back extensor strength were measured in the sitting position, followed by those measured standing, while the lowest values were measured in the prone position. No other studies have compared lower back extensor strength in different postural positions. Some studies have suggested that it is reasonable 
to use pelvic fixation [12] and, at the same time, a semi-sitting position with a hip flexion angle of $40-60^{\circ}$, since this position appears to increase the specificity of back extensor action (contraction) and reduce hip extensor activity [12,23]. In sitting and standing positions, this study employed pelvic fixation to specify the movement of the back extensor muscles, as suggested above $[17,18,23]$. However, the current study did not reach the semisitting position (40-60 $)$; it prioritized simplicity, requiring less time- and space-consuming procedures; creating a chair that could achieve the necessary angles for every subject would have added to study difficulty.

On the other hand, the chair deployed herein provided similar circumstances for all patients and measurements. The contraction of lumbar extensors in this position still appeared to be the strongest out of all three positions. The anchor rails required for measurements in the standing position are even cheaper than the specially designed chair and less space-consuming. However, they are not easy to move, so the measures always need to be taken in the same room. The technique for the prone position requires no special equipment (except the HHD itself).

While using an HHD, it may be difficult for the assessor to provide a counter-pressure that corresponds to the effort exerted by the patient, especially when measuring larger muscle groups [24]. This raises a question regarding the assessment of isometric lower back extensor strength using an HHD in the prone position, as the maximal strength of the examiner may limit it, and a ceiling effect may occur when testing strong individuals $[15,19,20]$. Valentine et al. suggest an alternative technique for prone position measurement, using a tripod fixed by a non-elastic belt to the table and the patient. The portable dynamometer is held between these [19]. External fixation can reduce measurement errors compared to assessments in which the assessor fixes the HHD manually. The Valentine et al. study results showed that, when using an HHD to assess lower back extensor strength, an HHD fixed with a tripod was superior to one that was manually set [19].

On the other hand, an ICC of $\geq 0.90$ is required for clinical application to ensure valid interpretation of any findings [25]; this study achieved this figure from the prone position without using tripod fixation. However, it must be borne in mind that movement in the prone position alone does not comply with the biomechanics of everyday life for most people. It may be anticipated that this movement could lead to discomfort in patients with low back pain and older people. Some of the studies even point out that several of their patients, those with hyperkyphosis due to osteoporotic fractures, were unable to perform the task [19].

The outcomes herein indicated that isometric lower back extensor strength in the prone position was mainly dependent on age (decreasing with advancing age). However, the isometric back extensor strength in sitting and standing positions seemed to be dependent not only on age, but predominantly on sex and weight; in the study population, weight was related to sex (data are not displayed); however, it can be assumed that the same pattern may also be seen in other populations of volunteers/patients. This finding accords with observations made by other authors [16], in which males exhibited higher extensor strength than females. Some authors explain this finding in terms of more excellent anthropometric dimensions and more elevated experience of maximum effort in men, which may allow them to achieve higher forces [16,26]. Mannion et al. posit that absolute muscle strength is determined by the quantity of force-generating elements and their geometry, so the strength of a muscle is mainly determined by its overall cross-sectional area [4]. The difference in maximum strength between the sexes may thus be simply associated with the gross muscle-size differences between men and women, a factor present in almost all skeletal muscles.

The current study disclosed a robust and significant correlation between strengthtesting results using HHD from the sitting and standing positions. Respiratory muscle strength proved a significant moderate correlation with isometric back extensor strength in sitting and standing positions, which could confirm validity for the technique measuring the lower back extensor muscle strength in sitting and standing positions using HHD. 
However, outcomes obtained from the prone position did not correlate strongly with those obtained from the other two positions. This did not hold for the technique performed in the prone position since the results did not correlate with respiratory muscle strength at all. These findings suggest to other working groups, who aim to assess lower back extensor strength using HHD in their practice, that one of the positions may be chosen to assess lower back extensor strength, preferably in a sitting or standing position, depending on the availability of equipment. In further works, the authors aim to use all these tests to observe the performance of patients with LBP and NMD; how do these results correlate and how do they differ from healthy volunteers. Only then will it be possible to exclude tests that seem to be redundant for clinical practice.

Respiratory muscles are an essential component of the core system. The strong correlation between maximal inspiratory pressure and maximal expiratory pressure that appeared in the results herein may support the validity of the techniques chosen for measuring respiratory muscle strength. In this study group, even though the volunteers tested were healthy, the overall strength of respiratory muscles in different subgroups did not exceed $100 \%$ of the predicted value. This may arise from the strict norms chosen, as they respect sex and age and find widespread application in this specialty [27]. In light of the fact that even asymptomatic people fail to reach predicted values, Caruso et al. presented a study that employed lower limits for the norms of respiratory muscle-strength tests: MIP $60 \mathrm{cmH}_{2} \mathrm{O}$ in women and $80 \mathrm{cmH}_{2} \mathrm{O}$ in men and MEP $120 \mathrm{cmH}_{2} \mathrm{O}$ and $150 \mathrm{cmH}_{2} \mathrm{O}$ in women and men, respectively [28]. All the study subgroups herein achieved their mean value for the lower limit of normal strength, except the subgroup of the youngest women, who achieved MEP values closely under this limit. It appears necessary to evaluate respiratory muscle strength since few studies point out possible discrepancies between the strength of the respiratory muscles and lumbar extensor muscles in patients with chronic LBP and patients with neuromuscular diseases [6,29-31].

The muscular endurance of the back extensor muscles was assessed herein by including the Biering-Sørensen test into the battery. This examination is widely accepted as a good assessment tool and predictor of non-specific low back pain [32,33]. The validity and repeatability of the test have been demonstrated in many studies [33-35]. The test examines the muscular endurance of all muscles involved in trunk extension, thus the paraspinal muscles (most notably the multifidus muscles) but also the hip extensors [33]. However, in the study baseline examination, the neurologist performed a manual muscle test of the lower extremities in all participants to ensure that no weakness was present and that the testing results would not be affected by potential weakness of the hip extensors.

In the group observed herein, a significant difference between the time of effort in men and women appeared in the Biering-Sørensen test. Women were able to hold the position for a longer time than men, which confirms the findings of other authors $[32,33]$. Mannion et al. explain the difference between the performances of the sexes in terms of the pronounced differences in the size ratios of the fiber types in the thoracic and lumbar regions of the erector spinae between them [4]. A significantly more extensive area of the muscle occupied by type 1 fibers (slow twitch) is found in the erector spinae of women. It seems that the greater the relative area of the muscles occupied by type 1 fibers, the longer the contraction can be sustained. In the groups observed herein, time of effort was lowest in volunteers under 30 years of age. The potential influence of age is not evident [33]. Some studies have taken the role of age into account, suggesting that back muscular endurance capacity may increase slightly with age [36,37]; others have contradicted this [32]. However, the study group herein was too small to conclude the matter.

Other trunk muscular endurance tests, the prone-plank test and side-bridge tests on both sides, were included in the study herein because the endurance imbalances between trunk muscle groups may also be related to low back disorders [38]. The prone-plank test has frequently been used in clinical settings to evaluate core stability, specifically abdominal core muscular endurance [14,39-41]. It is often regarded as the trunk flexor endurance test since the internal oblique, external oblique, and transverse abdominis muscles are largely 
activated during the procedure $[13,42]$. The side-bridge test is also an assessment that challenges core muscles, especially the lateral stabilizers [14,43]. These positions are also widely used as core stabilization exercise tools $[38,42,44,45]$. All the muscular endurance tests above have confirmed their validity and reliability in populations of athletes and healthy volunteers in previous studies $[41,43,46]$. They have been used in patients with LBP to assess trunk function $[40,42,47]$.

In contrast to the results of the Biering-Sørensen test, the prone-plank test in this study revealed no significant differences in time of effort among either age groups or the sexes. However, more effort was observed in men than in women, although they did not achieve statistical significance. Comparing the results herein with those of a study targeted on norms for the prone plank-test [48]. The study data were more similar to the athletic part of the previously studied population. This fact might well be explained by the high familiarity of the exercise position (prone-plank position) in socioeconomic relationships. In contrast to the Biering-Sørensen test, where none of the volunteers had ever seen or tried the position, the prone-plank exercise was widely known, and only two of the volunteers had never seen or tried it before.

Outcomes obtained in the side-bridge test showed a robust correlation between right and left sides, so it might be suggested that the test be performed on only one side. No difference in outcomes of this test appeared between the sexes in the current study. However, significant differences between age groups were observed for the right side only, a trend that may arise out of the cohort being relatively small. Evans et al., who tested a group of young athletes using the side-bridge test, found performances differed between the sexes on both sides [46]. However, a significant difference in muscular endurance performance between sex and age groups in some muscle groups but not others implies that trunk muscular endurance should be tested comprehensively. The side-bridge test should be performed on both sides [46].

The outcomes of the two trunk muscular endurance tests, the Biering-Sørensen and prone-plank tests, correlated, albeit only moderately. Most importantly, this study revealed a different distribution model of outcomes between age groups and the sexes in these two tests. The correlation between the side-bridge test and the prone-plank test was again moderate. It appears that all of these tests are predictive of core muscular endurance, but it is apparent that each test examines different muscle co-activations, determined by the postural situation. It therefore appears reasonable to include all three trunk muscular endurance examinations in the final test battery.

The correlation between strength-testing procedures and trunk muscular endurance tests was weak or absent. This finding suggests that there might be discrepancies between absolute strength and muscular endurance of trunk muscles even in asymptomatic people; both of these aspects should therefore be observed. Both isometric lower back extensor strength and trunk muscular endurance tests should be included in the final test battery.

In the study group herein, outcomes for strength and muscular endurance tests were not dependent on participants' general physical activity levels. The findings of specific authors suggest that the level of general physical activity does not predict or initiate LBP; however, well-chosen measures may reveal deconditioning of certain muscle groups associated with LBP [12]. It remains crucial to monitor the activeness of patients, as specialists in the field constantly address this issue.

In further studies, the authors of the current contribution intend to deploy this battery of tests as a whole in patients with low back pain and neuromuscular diseases to verify whether any of these outcome measures are able to recognize altered trunk muscle function and trunk muscle imbalance in such disorders. However, while using these tests in different groups of patients, the possibility of pain elicited throughout the testing procedures must be recorded and taken into account while interpreting the results. We anticipate that these tests will be helpful in clinical practice. They have the potential to capture changes in outcomes concerning disease progression or interventions, such as rehabilitation programs aiming for improvement in the strength and muscular endurance of the lumbar extensor 
muscles and/or core muscles. However, as mentioned above, further studies need to be conducted to prove these assumptions.

\section{Limitations of the Study}

The current study has its limitations. Firstly, only 60 volunteers were enrolled, so individual age sub-groups could not consist of more than 15 people. Normative data for each test were therefore impossible to achieve. However, the study provides an overview of the trunk muscle strengths and muscular endurance performances that might be expected in the healthy population's various age and sex groups in the socioeconomic background. Secondly, all participants were hospital employees, so this was not a heterogeneous group of people according to education, as $80 \%$ of volunteers had graduated from university.

On the other hand, heterogeneity existed in terms of the nature of their work (sedentary/manual). Thirdly, only intra-rater reliability of strength testing procedures using HHD was established, as there was only one experienced examiner available for this study. Therefore, one should be aware of the possibility of a "ceiling effect" that may occur in powerful individuals when isometric lower back extensor strength is measured using an HHD in the prone position. Another limitation of the study is that we did not record pain throughout the assessment. This might be because exclusion criteria were defined as existing acute and chronic low back pain. It needs to be pointed out that all volunteers were highly motivated to participate in the study and did not complain concerning any pain throughout the assessments. While there is a possibility that pain elicited during the test may interfere with measurements, especially in patients with low back pain, this needs to be recorded, taken into account, and considered in the interpretation of the results. Further studies could include pain-detecting protocols and questionnaires at the enrollment and during the procedures to record whether any tests trigger pain.

\section{Conclusions}

This study has defined and unified the methodology of a simple test battery that provides a comprehensive picture of trunk-muscle function (strength and endurance), including the lumbar extensors. All the tests herein are easy to perform in everyday clinical practice, are reasonable in terms of time and cost, and, at the same time, are reliable and valid. The results of this study may contribute to a better understanding of the lumbar extensor muscles because an essential first step in diagnosis is understanding the natural patterns of performance in the healthy population.

Author Contributions: Conceptualization, D.V., P.K. and B.A.; methodology, D.V. and B.A.; software, P.K. and M.K.; validation, D.V., P.K. and M.K.; formal analysis, M.K. and E.V.; investigation, D.V. and P.K.; resources, D.V.; data curation, D.V., P.K., E.V., M.K. and B.A.; writing-original draft preparation, D.V. and B.A.; writing-review and editing, L.B., F.D. and S.V.; visualization, P.K. and L.B.; supervision, B.A.; project administration, S.V.; funding acquisition, S.V. All authors have read and agreed to the published version of the manuscript.

Funding: This work was supported by the Ministry of Health of the Czech Republic project for conceptual development in research organizations, ref. no. 65269705 (University Hospital Brno, Brno, Czech Republic). Five authors of this publication are members of the European Reference Network for Neuromuscular Diseases-Project ID no. 870177.

Institutional Review Board statement: The study was conducted according to the guidelines of the Declaration of Helsinki, and approved by the Ethics Committee in each institution.

Informed Consent statement: Informed consent was obtained from all subjects involved in the study.

Data Availability Statement: The data presented in this study are available on request from the corresponding author. The data are not publicly available.

Conflicts of Interest: The authors declare no conflict of interest. 


\section{References}

1. Daggfeldt, K.; Huang, Q.-M.; Thorstensson, A. The visible human anatomy of the lumbar erector spinae. Spine 2000, 25, 2719-2725. [CrossRef] [PubMed]

2. Crawford, R.J.; Cornwall, J.; Abbott, R.; Elliott, J.M. Manually defining regions of interest when quantifying paravertebral muscles fatty infiltration from axial magnetic resonance imaging: A proposed method for the lumbar spine with anatomical cross-reference. BMC Musculoskelet. Disord. 2017, 18, 25. [CrossRef] [PubMed]

3. Agten, A.; Verbrugghe, J.; Stevens, S.; Boomgaert, L.; O Eijnde, B.; Timmermans, A.; Vandenabeele, F. Feasibility, accuracy and safety of a percutaneous fine-needle biopsy technique to obtain qualitative muscle samples of the lumbar multifidus and erector spinae muscle in persons with low back pain. J. Anat. 2018, 233, 542-551. [CrossRef] [PubMed]

4. Mannion, A.F. Fibre type characteristics and function of the human paraspinal muscles: Normal values and changes in association with low back pain. J. Electromyogr. Kinesiol. 1999, 9, 363-377. [CrossRef]

5. Adams, M.A.; Hutton, W.C. Has the lumbar spine a margin of safety in forward bending? Clin. Biomech. 1986, 1, 3-6. [CrossRef]

6. Kolář, P.; Šulc, J.; Kynčl, M.; Šanda, J.; Čakrt, O.; Andel, R.; Kumagai, K.; Kobesová, A. Postural function of the diaphragm in persons with and without chronic low back pain. J. Orthop. Sports Phys. Ther. 2012, 42, 352-362. [CrossRef] [PubMed]

7. Kalichman, L.; Carmeli, E.; Been, E. The association between imaging parameters of the paraspinal muscles, spinal degeneration, and low back pain. BioMed Res. Int. 2017, 2017, 2562957. [CrossRef]

8. Hildebrandt, M.; Fankhauser, G.; Meichtry, A.; Luomajoki, H. Correlation between lumbar dysfunction and fat infiltration in lumbar multifidus muscles in patients with low back pain. BMC Musculoskelet. Disord. 2017, 18, 12. [CrossRef]

9. Freeman, M.D.; Woodham, M.A.; Woodham, A.W. The role of the lumbar multifidus in chronic low back pain: A review. PMER 2010, 2, 142-146. [CrossRef]

10. Padwal, J.; Berry, D.B.; Hubbard, J.C.; Zlomislic, V.; Allen, R.T.; Garfin, S.R.; Ward, S.R.; Shahidi, B. Regional differences between superficial and deep lumbar multifidus in patients with chronic lumbar spine pathology. BMC Musculoskelet. Disord. 2020, 21, 764. [CrossRef]

11. Steele, J.; Fisher, J.; Perrin, C.; Conway, R.; Bruce-Low, S.; Smith, D. Does change in isolated lumbar extensor muscle function correlate with good clinical outcome? A secondary analysis of data on change in isolated lumbar extension strength, pain, and disability in chronic low back pain. Disabil. Rehabil. 2018, 41, 1287-1295. [CrossRef] [PubMed]

12. Steele, J.; Bruce-Low, S.; Smith, D. A reappraisal of the deconditioning hypothesis in low back pain: Review of evidence from a triumvirate of research methods on specific lumbar extensor deconditioning. Curr. Med Res. Opin. 2014, 30, 865-911. [CrossRef] [PubMed]

13. Imai, A.; Kaneoka, K. The relationship between trunk endurance plank tests and athletic performance tests in adolescent soccer players. Int. J. Sports Phys. Ther. 2016, 11, 718-724.

14. Avers, D.; Brown, M. Daniels and Worthingham's Muscle Testing: Techniques of Manual Examination and Performance Testing; Elsevier: Philadelphia, PA, USA, 2018; pp. 48-80.

15. Witting, N.; Andersen, L.K.; Vissing, J. Axial myopathy: An overlooked feature of muscle diseases. Brain 2016, 139, 13-22. [CrossRef]

16. García-Vaquero, M.P.; Barbado, D.; Juan-Recio, C.; López-Valenciano, A.; Vera-Garcia, F.J. Isokinetic trunk flexion-extension protocol to assess trunk muscle strength and endurance: Reliability, learning effect, and sex differences. J. Sport Health Sci. 2020, 9 , 692-701. [CrossRef]

17. Harding, A.T.; Weeks, B.K.; Horan, S.A.; Little, A.; Watson, S.L.; Beck, B.R. Validity and test-retest reliability of a novel simple back extensor muscle strength test. SAGE Open Med. 2017, 5, 2050312116688842. [CrossRef]

18. Park, H.-W.; Baek, S.; Kim, H.Y.; Park, J.-G.; Kang, E.K. Reliability and validity of a new method for isometric back extensor strength evaluation using a hand-held dynamometer. Ann. Rehabil. Med. 2017, 41, 793-800. [CrossRef] [PubMed]

19. Valentin, G.; Maribo, T. Hand-held dynamometry fixated with a tripod is reliable for assessment of back extensor strength in women with osteoporosis. Osteoporos. Int. 2014, 25, 2143-2149. [CrossRef]

20. Sigmund, E.; Zacpal, J.; Sigmundová, D.; Mitáš, J.; Sklenářr, V.; Bělohlávek, R.; Frömel, K. Evaluation of IPAQ questionnaires using the formal concept analysis. Stud. Kinanthropol. 2007, 8, 7-16. [CrossRef]

21. Guidelines for Data Processing and Analysis of the International Physical Activity Questionnaire (IPAQ)-Short and Long Forms, Revised November 2005. Available online: https:/ / sites.google.com/site/theipaq/scoring-protocol (accessed on 4 November 2010).

22. Evans, J.D. Straightforward Statistics for the Behavioral Sciences; Brooks/Cole Pub. Co.: Pacific Grove, CA, USA, 1996.

23. Da Silva, R.A.; Lariviere, C.; Arsenault, A.B.; Nadeau, S.; Plamondon, A. Pelvic stabilization and semisitting position increase the specificity of back exercises. Med. Sci. Sports Exerc. 2009, 41, 435-443. [CrossRef]

24. Kolber, M.J.; Cleland, J.A. Strength testing using hand-held dynamometry. Phys. Ther. Rev. 2005, 10, 99-112. [CrossRef]

25. Innes, E.; Straker, L. Reliability of work-related assessments. Work 1999, 13, 107-124. [PubMed]

26. Keller, A.; Hellesnes, J.; Brox, A.J.I. Reliability of the isokinetic trunk extensor test, biering-sørensen test, and Åstrand bicycle test: Assessment of intraclass correlation coefficient and critical difference in patients with chronic low back pain and healthy individuals. Spine 2001, 26, 771-777. [CrossRef] [PubMed]

27. Black, L.F.; Hyatt, R.E. Maximal respiratory pressures: Normal values and relationship to age and sex. Am. Rev. Respir. Dis. 1969, 99, 696-702. [CrossRef] 
28. Caruso, P.; Albuquerque, A.L.; Santana, P.V.; Cardenas, L.Z.; Ferreira, J.G.; Prina, E.; Trevizan, P.F.; Pereira, M.C.; Iamonti, V.; Pletsch, R.; et al. Diagnostic methods to assess inspiratory and expiratory muscle strength. J. Bras. Pneumol. 2015, 41, 110-123. [CrossRef]

29. Janssens, L.; Brumagne, S.; Polspoel, K.; Troosters, T.; McConnell, A. The effect of inspiratory muscles fatigue on postural control in people with and without recurrent low back pain. Spine 2010, 35, 1088-1094. [CrossRef]

30. Benditt, J.O. Pathophysiology of neuromuscular respiratory diseases. Clin. Chest Med. 2018, 39, 297-308. [CrossRef] [PubMed]

31. Finta, R.; Nagy, E.; Bender, T. The effect of diaphragm training on lumbar stabilizer muscles: A new concept for improving segmental stability in the case of low back pain. J. Pain Res. 2018, 11, 3031-3045. [CrossRef]

32. Biering-Sørensen, F. Physical measurements as risk indicators for low-back trouble over a one-year period. Spine 1984, 9, 106-119. [CrossRef]

33. Demoulin, C.; Vanderthommen, M.; Duysens, C.; Crielaard, J.-M. Spinal muscle evaluation using the Sorensen test: A critical appraisal of the literature. Jt. Bone Spine 2006, 73, 43-50. [CrossRef]

34. Denteneer, L.; Van Daele, U.; Truijen, S.; De Hertogh, W.; Meirte, J.; Stassijns, G. Reliability of physical functioning tests in patients with low back pain: A systematic review. Spine J. 2018, 18, 190-207. [CrossRef]

35. Martínez-Romero, M.T.; Ayala, F.; De Ste Croix, M.; Vera-Garcia, F.J.; Sainz de Baranda, P.; Santonja-Medina, F.; Sánchez-Meca, J. Meta-analysis of the reliability of four field-based trunk extension endurance tests. Int. J. Environ. Res. Public Health 2020, 17, 3088. [CrossRef] [PubMed]

36. Kankaanpää, M.; Laaksonen, D.; Taimela, S.; Kokko, S.-M.; Airaksinen, O.; Hänninen, O. Age, sex, and body mass index as determinants of back and hip extensor fatigue in the isometric Sørensen back endurance test. Arch. Phys. Med. Rehabil. 1998, 79, 1069-1075. [CrossRef]

37. Yassierli; Nussbaum, M.A.; Iridiastadi, H.; Wojcik, L.A. The influence of age on isometric endurance and fatigue is muscle dependent: A study of shoulder abduction and torso extension. Ergonomics 2007, 50, 26-45. [CrossRef]

38. Juan-Recio, C.; López-Plaza, D.; Barbado Murillo, D.; García-Vaquero, M.P.; Vera-García, F.J. Reliability assessment and correlation analysis of 3 protocols to measure trunk muscle strength and endurance. J. Sports Sci. 2018, 36, 357-364. [CrossRef] [PubMed]

39. Ekstrom, R.A.; Donatelli, R.A.; Carp, K.C. Electromyographic analysis of core trunk, hip, and thigh muscles during 9 rehabilitation exercises. J. Orthop. Sports Phys. Ther. 2007, 37, 754-762. [CrossRef]

40. Schellenberg, K.L.; Lang, J.M.; Chan, K.M.; Burnham, R.S. A clinical tool for office assessment of lumbar spine stabilization endurance: Prone and supine bridge maneuvers. Am. J. Phys. Med. Rehabil. 2007, 86, 380-386. [CrossRef]

41. De Blaiser, C.; De Ridder, R.; Willems, T.; Danneels, L.; Vanden Bossche, L.; Palmans, T.; Roosen, P. Evaluating abdominal core muscle fatigue: Assessment of the validity and reliability of the prone bridging test. Scand. J. Med. Sci. Sports 2017, 28, 391-399. [CrossRef]

42. Kong, Y.-S.; Park, S.; Kweon, M.-G.; Park, J.-W. Change in trunk muscle activities with prone bridge exercise in patients with chronic low back pain. J. Phys. Ther. Sci. 2016, 28, 264-268. [CrossRef]

43. McGill, S.M.; Childs, A.; Liebenson, C. Endurance times for low back stabilization exercises: Clinical targets for testing and training from a normal database. Arch. Phys. Med. Rehabil. 1999, 80, 941-944. [CrossRef]

44. Ekstrom, R.A.; Osborn, R.W.; Hauer, P.L. Surface electromyographic analysis of the low back muscles during rehabilitation exercises. J. Orthop. Sports Phys. Ther. 2008, 38, 736-745. [CrossRef]

45. Ozsoy, G.; Ilcin, N.; Ozsoy, I.; Gurpinar, B.; Buyukturan, O.; Buyukturan, B.; Kararti, C.; Sas, S. The effects of myofascial release technique combined with core stabilization exercise in elderly with non-specific low back pain: A randomized controlled, single-blind study. Clin. Interv. Aging 2019, 14, 1729-1740. [CrossRef] [PubMed]

46. Evans, K.; Refshauge, K.M.; Adams, R. Trunk muscle endurance tests: Reliability, and gender differences in athletes. J. Sci. Med. Sport 2007, 10, 447-455. [CrossRef] [PubMed]

47. Vanti, C.; Conti, C.; Faresin, F.; Ferrari, S.; Piccarreta, R. The relationship between clinical instability and endurance tests, pain, and disability in nonspecific low back pain. J. Manip. Physiol. Ther. 2016, 39, 359-368. [CrossRef] [PubMed]

48. Strand, S.L.; Hjelm, J.; Shoepe, T.C.; Fajardo, M.A. Norms for an isometric muscle endurance test. J. Hum. Kinet. 2014, 40, 93-102. [CrossRef] [PubMed] 